\title{
Evaluation of anti-hepatotoxic potential of Cassia javanica (L.) methanol extract against paracetamol- induced liver damage in Wistar albino rats
}

\author{
Esther Lalhmingliani, Zodinpuii, R.K. Lalremtluangi, Lalramdinthara Chenkual, \\ Lalthansangi
}

Department of Zoology, Mizoram University, Tanhril 796004, Mizoram, India

Corresponding author: esther19ralte@gmail.com

\begin{abstract}
Species of Cassia (family Caesalpinaceae) are well known in India and other tropical countries for their medicinal values. This study evaluates the efficacy of the methanol extract of the leaves of Cassia javanica $L$. on paracetamol-induced liver damage in experimental rats. The animals were randomly divided into six groups of five animals each. While group I served as the normal control, group II-V serve as the test groups, and group VI as C javanica control group. Hepatotoxicity was induced in the test groups via oral administration of paracetamol ( $2 \mathrm{~g} / \mathrm{kg} \mathrm{b}$.wt). While group III-V were treated for 14 days with $125 \mathrm{mg} / \mathrm{kg}, 250$ $\mathrm{mg} / \mathrm{kg}$ and $500 \mathrm{mg} / \mathrm{kg}$ b.wt of C javanica methanol extract respectively, group II served as positive control and was left untreated. The degree of hepatoprotection was assessed by estimating the levels of aspartate transaminases (AST), alanine aminotransferase (ALT), alkaline phosphatase (ALP), total bilirubin and tissue protein. Administration of methanol extract of $C$ javanica elicited protective action in groups III-V when compared to group II as the cell progress towards normalization evidenced by the significant decline in the elevated levels of marker enzymes (AST, ALT, ALP) and total bilirubin and an increase in tissue protein. The findings indicate that $\mathrm{C}$ javanica has a hepatoprotective action against paracetamol-induced hepatic damage in Wistar rats and indeed validate its traditional use in the treatment of liver damage.
\end{abstract}

Keywords: Cassia javanica, hepatoprotection, liver damage, paracetamol.

\section{INTRODUCTION}

Liver is a vital organ playing a pivotal role in intermediary metabolism and regulating various physiological processes in the body (Mayuren et al., 2010). It is involved with many biochemical pathways related to growth, defence, nutrient supply, energy provision, reproduction and is the frequent target of a number of toxicants (Ward et al., 1999; Meyer et al., 2001). Liver diseases are mainly caused by toxic chemicals, excess consumption of alcohol, infections, environmental pollutants drugs and autoimmune disorders. Most hepatotoxic agents damage liver cells through induction of lipid peroxidation and other oxidative damages (Boyh and Berec$z k y, 1966)$. These chemical agents impose excessive stress on the liver filtering function and liver damage may occur if the toxins accumulate faster than the me- tabolizing activity of the liver (Bigoniya et al., 2009).

Nature has provided a source of new drugs for thousands of years and an impressive amount have been isolated from natural sources, based mainly on their use in folklore medicine (Usha et al., 2012). Cassia species (Caesalpinaceae) are well known medicinal plant commonly found in India and other tropical countries. The extract of Cassia species leaves has been found to possess significant hepatoprotective activity and antiinflammatory activity (Manojlovic et al., 2006).

Paracetamol is believed to be safe, having analgesic and antipyretic effects at therapeutic doses and unlike other drugs, it has only weak anti-inflammatory properties (Prescott and Critchley, 1983; Bessems and Vermeulen 2001). Although considered safe, at higher doses it produces centrilobular hepatic necrosis that can be fatal (Proudfoot and Wright, 1970; Prescott, 1980; Cob- 
den et al., 1982). Liver damage may be detected as raised serum transaminases, viz. aspartate transaminase (AST) and alanine aminotransferase (ALT) which may reach levels of $5000 \mathrm{IU} / \mathrm{L}$ (Prescott et al., 1971). However, bilirubin levels may be only moderately elevated. This study examines the effect of Cassia javanica on the paracetamol-induced hepatic damage in rats.

\section{MATERIALS AND MeTHOdS}

\section{Preparation of plant extract}

The leaves of $\mathrm{C}$. javanica were collected from Electric Veng, Lunglei, Mizoram. It was identified and authenticated by taxonomist at the Department of Botany, Mizoram University. The leaves were washed and allowed to shade dry at room temperature in clean and hygienic conditions and powdered using an electrical grinder. Air-dried and finely powdered leaves was then Soxhlet extracted with methanol, filtered using Whatman filter paper no. 1 and evaporated to dryness in an oven at $40^{\circ} \mathrm{C}$. A dark brown extract was obtained and stored at $-20^{\circ} \mathrm{C}$ until further use. The extract was then dissolved in normal saline and given orally to the animal.

\section{Animal model and ethical clearance}

Adult Wistar albino rats of both sexes (weighing 150 $200 \mathrm{~g}$ ) were housed in polypropylene cages containing sterile paddy husk (procured locally) as bedding and allowed to acclimatize to the laboratory environment at Animal Care Facility at the Department of Zoology, Mizoram University, Aizawl, India for 2 weeks. The animals were fed with commercially available food pellets and water ad libitum. Ethical clearance was obtained from the Institutional Animal Ethical Committee, Mizoram University (MZU-IAEC/2018/05).

\section{Acute toxicity study}

Acute toxicity study was performed as per Organization for Economic Co-operative and development (OECD) guidelines No. 423.8 (OECD, 2000). The animals were kept fasting for overnight before the treatment. C. javanica methanol extract was then administered orally as single dose. The animals were first observed individually during the first 30 minutes and periodically during 24 hours with special attention given during the first 4 hours and daily thereafter, for a total of 14 days. Administration of $5 \mathrm{~g} / \mathrm{kg}$ b.wt has no toxicity as no mortality was observed for 14 days.

\section{Induction of hepatic injury}

Hepatic damage was induced in experimental animals by oral administration of paracetamol ( $2 \mathrm{~g} / \mathrm{kg}$ b.wt) daily for 14 days. Paracetamol was procured from local drug store and was dissolved in $2 \mathrm{ml}$ normal saline. Biochemical assay kits and all other reagents used in the study were of analytical grade.

\section{Hepato-protective test}

Experimental animals were randomized into six groups with five animals in each group. Group I animals were administered a single daily dose of normal saline $(200 \mu \mathrm{l})$ and served as normal control. All the animals of group II $-\mathrm{V}$ received paracetamol $2 \mathrm{~g} / \mathrm{kg}$, b.wt. While group III-V were treated with $125 \mathrm{mg} / \mathrm{kg}, 250 \mathrm{mg} / \mathrm{kg}$ and $500 \mathrm{mg} / \mathrm{kgb}$.wt of C. javanica methanol extract respectively, group II served as positive control and was left untreated. Group VI animals served as C. javanica control and received $C$. javanica extract only. Treatment was carried out orally for 14 consecutive days.

On the $15^{\text {th }}$ day all animals were sacrificed by cervical dislocation and blood samples and livers were collected. The liver was cleared off of extraneous materials and the weight was noted down. Blood was left undisturbed for 10 minutes. The clot was then dispersed with glass rod and centrifuged at $10000 \mathrm{rpm}$ for 5 minutes to separate the serum which was subsequently analyzed for various biochemical parameters including aspartate aminotransferases (AST), alanine aminotransferases (ALT), alkaline phosphatase (ALP) and bilirubin by using commercial kits as per the instructions. The estimation of AST and ALT followed the method of Reitman and Frankel's (1957). Kind and Kings (1954) method was followed for ALP estimation and for bilirubin estimation, Jendrassik and Grof's method (1938) were followed. Potein was estimated according to the method of Lowry et al. (1951).

Statistical analysis was performed using one-way ANOVA followed by Tukey multiple comparison of means. The values were expressed as mean \pm SEM $(n=5)$. The analysis was carried out using SPSS ver. 20 . The value $p<0.05$ was considered to be statistically significant.

\section{RESULTS AND DISCUSSIONS}

Paracetamol treatment leads to significant increase in the level of serum biomarker enzymes (AST, ALT and ALP) in positive control group when compared to normal control $(p<0.003)$. Dose-dependent treatment with $C$. javanica methanol extract significantly brought back the altered serum marker enzymes levels to near normal against paracetamol treated rats indicating protection against toxin except in group III (Figure 1a-c). The elevated levels of these biomarker enzymes are direct reflection of alterations in the hepatic structural integrity, an indicative of cellular leakage and loss of functional integrity in liver (Drotman, 1978). In particular, the increase in the level of serum ALT is indicative of liver damage. These enzymes are located in the cell cytoplasm and are emptied into the circulation once the cellular mem- 


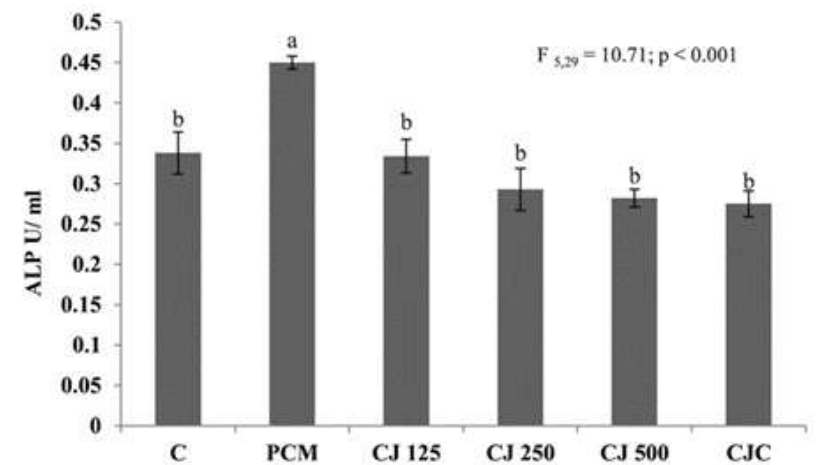

(a)

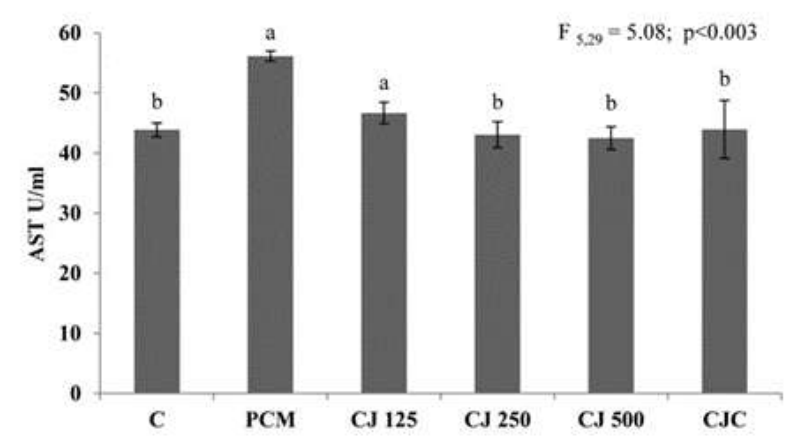

(c)

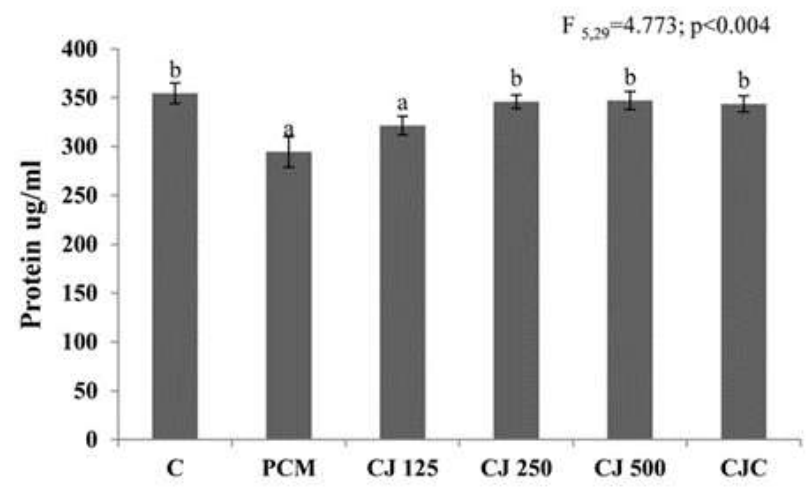

(e)

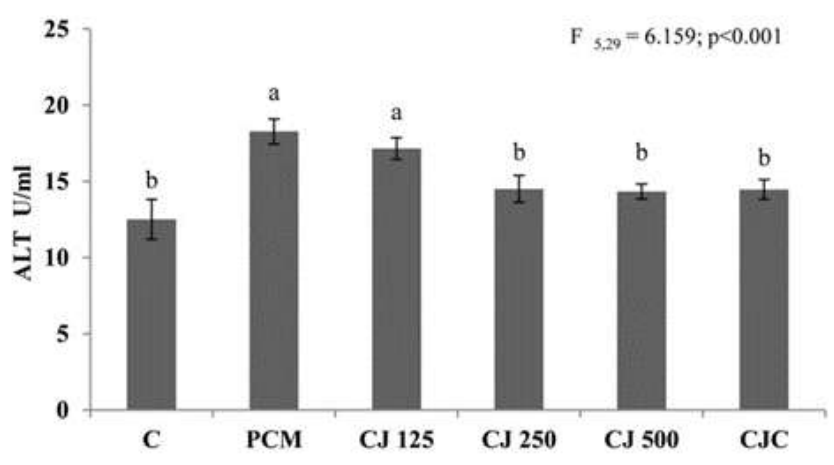

(b)

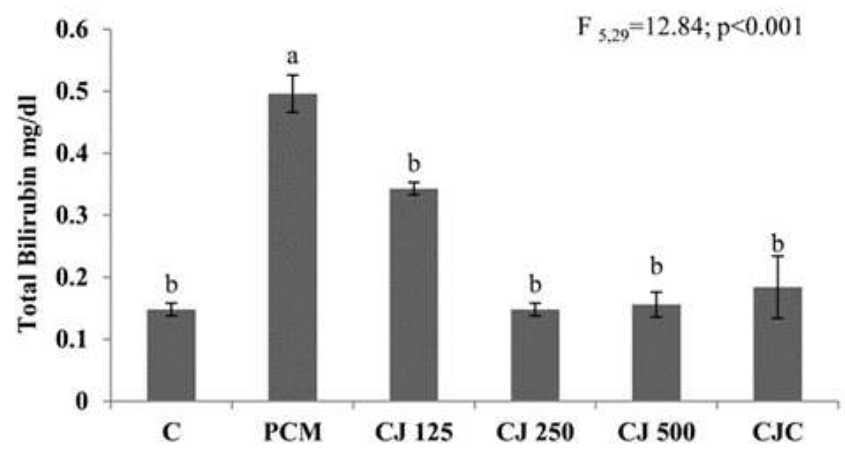

(d)

Figure 1: Effect of $C$. javanica methanol extract on paracetamol induced liver damage in rat. (a) ALP (U/ml); (b) ALT $(\mathrm{U} / \mathrm{ml}) ;(\mathrm{c})$. AST $(\mathrm{U} / \mathrm{ml})$; (d) Total bilirubin $(\mathrm{mg} / \mathrm{dl}) ;(\mathrm{e})$ Protein $(\mu \mathrm{g} / \mathrm{ml})$. Means not sharing the same letter are significantly different. C-Normal control group; PCM-paracetamol treated group; CJ125, 250 \& 500 - C. javanica extract treated group $(125,250$ and $500 \mathrm{mg} / \mathrm{kg}$ b.wt) respectively with paracetamol treatment $(2 \mathrm{gm} / \mathrm{kg})$; CJC- C. javanica control group. 
brane is damaged (Lin et al., 2000). A pronounced elevation in the concentration of bilirubin in response to paracetamol intoxication also exhibit a significant decline with dose dependent treatment of $C$. javanica methanol extract $(p<0.001)$. The decrease in extract treated group is almost comparable with the positive control group (Figure 1d.).

This observation is in accordance with the work of Sivakumar et al. (2014) on Solanum xanthocarpum in paracetamol induced hepatic damage in rats and Xanvier et al. (2014) on Centella asiatica in $\mathrm{CCl}_{4}$-induced liver injury in rats. The elevation of marker enzymes in rats administered with paracetamol reported in this study is also in line with the findings of Prakash (2008). On the other hand, treatment with paracetamol leads to significant decrease $(p<0.004)$ in the level of protein in the positive control group as compared to normal control. However, a significant increase in protein, almost comparable to the normal control was observed in the extract treated animals except in group III (Figuren1e). The results in this study indicated that treatment of experimental animals for 14 days with C. javanica extract at varying dosages to paracetamol administration significantly reversed the changes and further demonstrated that the effects of toxicity induced by paracetamol on the liver function could be effectively counterbalanced by treatment with $C$. javanica methanol extract. The efficacy of any hepatoprotective agent dwells on its ability to either sustain the normal hepatic architecture and physiological mechanism disturbed by a hepatotoxin or reduce the negative effects induced by hepatotoxic agent.

\section{REFERENCES}

Bonkovsky, H.L., Kane, RE., Jone, D.P., Galinsky, R.E., Banner, E. (1994). Acute hepatic and renal toxicity from low doses of acetaminophen in the absence of alcohol abuse or malnutrition: evidence for increased susceptibility to drug toxicity due to cardiopulmonary and renal insufficiently. Hepatology, 19, 1141-1448.

Boyd, E.H., Bereczky, G.M. (1966). Liver necrosis from paracetamol. British Journal of pharmacology, 26, 606 $-614$.

Bessems, J.G., Vermeulen, N.P (2001). Paracetamol (acetaminophen)-induced toxicity: molecular and biochemical mechanisms, analogues and protective approaches. Critical Reviews in Toxicology, 31, 55138.

Bigoniya, P., Singh, C.S., Shukla, A. (2009). A comprehensive review of different liver toxicants used in experimental pharmacology. International Journal of Pharmacy and Pharmaceutical Sciences and Drug Research, 1, 124-135.

Cobden, I., Record, C.O., Ward, M.K., Kerr, D.N.S. (1982). Paracetamol induced acute renal failure in the $a b$ sence of fulminant liver damage. British Medical Journal, 284, 21-22.
Drotman, R.B., Lawhorn, G.T. (1978). Serum enzymes are indicators of chemical induced liver damage. Drug and Chemical Toxicology, 1, 163-171.

Jendrassik, L., Groff, P. (1938). Bilirubin kit. Biochemistry, 2297, 81.

Kind, P.R.N., King, E.J. (1954). Estimation of plasma phosphatase by determination of hydrolysed phenol with amino-antipyrine. Journal of clinical Pathology, 7(4), 322-326.

Lowry, O.H., Rosebrough, N.J., Randall, R.J. (1951). Protein measurement with the folin phenol reagent. Biochemistry, 193, 265-275.

Lin, H.Z., Yang, S.Q., Chuckaree, C., Kuhajda, F., Ronnet, G., Diehl, A.M. (2000). Metformin reverses fatty liver disease in obese, leptin-deficient mice. Natural Medicine 6, 998-1003.

Meyer, S.A., Kulkarni, A.P. (2001). Hepatotoxicity. Introduction to Biochemical Toxicology (3rd edition). New York: John Wiley \& Sons, pp. 486-487.

Manojlovid, I., Bogdanovid-Dusanovid, G., Gritsanapan, W., Manojlovid, N. (2006). Isolation and identification of anthraquinones of Caloplaca cerina and Cassia tora. Chemical Papers, 60(6), 466-468.

Mayuren, C., Reddy, V.V., Priya, S.V, Devi, V.A. (2010). Protective effect of livactin against $\mathrm{CCl}(4)$ and paracetamol induced hepatotoxicity in adult Wistar rats. North American Journal of Medical Sciences 2, 491-495.

Prakash., Satyan, K.S., Wahi, S.P., Singh, R.P. (1995). Comparative hepatoprotective activity of three Phyllanthus species, P. urinaria, P. niruri and P. simplex, on carbon tetrachloride induced liver injury in the rat. Phytotherapy Research, 9, 594-596.

Proudfoot, A.T., Wright, N. (1970). Acute paracetamol poisoning. British Medical Journal, 3, 557-558.

Prescott, L.F., Rosecoe, P., Wright, N., Brown, S.S. (1971). Plasma-paracetamol half-life and hepatic necrosis in patients with overdosage. The Lancet, 1, 519-522.

Prescott, L.F. (1980). Hepatotoxicity of mild analgesics. British Journal of Clinical Pharmacology, 10, 373-379.

Prescott, L.F., Critchley, J.A. (1983). The treatment of acetaminophen poisoning. Annual Review of Pharmacology and Toxicology, 23, 87-101.

Reitman, S., Frankel, S. (1957). A colorimetric method for the determination of serum glutamic oxalacetic and glutamic pyruvic transaminases. American Journal of Clinical Pathology, 28(1), 56-63.

Sivakumar, V., Dhanarajan, M.S. (1966). Antioxidant effect of Tinosporacordifolia extract in alloxan induced diabetic rats. Indian Journal of Pharmaceutical Sciences, 72(6), 79598.

Xanvier, S., Umadevi, D. (2014). Hepatoprotective effect of gotu kola (Centella asiatica Linn.) on carbon tetrachloride liver injury in rats. International Journal of Research Pharmacy, 5 (12), 929-931. 